\title{
Progettare la città storica, sussidiario minimo
}

Bertrando Bonfantini

Politecnico di Milano, Dipartimento di Architettura e Studi Urbani (bertrando.bonfantini@polimi.it)

Nel 2021 l'ANCSA, Associazione Nazionale Centri Storico-Artistici, compie sessant'anni. Questa nota lo ricorda con un testo particolare: una sorta di sussidiario minimo del progetto della città storica. ${ }^{1}$ Costituisce anche l'occasione per richiamare l'originale pubblicazione di 30 anni Ancsa 1960-1990, adesso che altri trenta sono trascorsi. ${ }^{2}$

1. Che fare della città 'ereditata' dal passato? È una questione che il progetto urbanistico non può eludere, almeno nei contesti di più antica urbanizzazione. In Italia, in Europa, questa è la storia di tre stagioni, e di altrettante differenti tematizzazioni: riformare la città vecchia, salvaguardare il centro storico, infrastrutturare la città contemporanea.

2. Il primo atteggiamento, fino agli anni '30 del secolo scorso, vede nella città del passato una città vecchia da sostituire: il progetto di riforma rimuove ciò che è visto di ostacolo al processo di modernizzazione urbana. Il secondo, con un cambio di prospettiva, si impone nel dibattito culturale del dopoguerra quando la nozione di centro storico viene a circoscrivere una intera parte della città, riconosciuta ora 'monumento unitario' da tutelare: il centro storico diventa isola pregiata da proteggere, ma anche spazio urbano 'sottratto'. Il terzo atteggiamento s'insinua dagli anni '80 del secolo scorso, quando cominciano a manifestarsi progetti che alla città ereditata attribuiscono un potenziale strutturante nella costruzione dell'urbanità contemporanea: la città storica diviene 'infrastruttura' che attraversa e innerva la compagine urbana e ne qualifica l'abitabilità.

3. Il progetto di riforma riconduce la città ereditata alle regole di funzionamento della città moderna. Adatta e omologa la città vecchia alla città nuova. L'intervento urbanistico opera una ridefinizione compositiva delle relazioni tra le parti, ri-strutturando l'assetto urbanistico preesistente. Lo spazio aperto - in particolare, lo spazio stradale, con risagomature e nuove aperture - è protagonista del progetto urbanistico e ne costituisce il cuore. Lo spazio edificato storico è materia malleabile, sacrificabile, disponibile alla trasformazione in funzione del nuovo assetto 'modernizzatore'.

4. L'allargamento delle sezioni viarie, le operazioni di rettifica stradale e nuovo allineamento dei fronti edilizi, l'apertura di nuovi tracciati con lo sventramento dei tessuti urbani esistenti, gli interventi di isolamento delle emergenze architettoniche e monumentali, e quelli di diradamento edilizio alimentano l'articolata gamma di procedimenti tecnici che si sedimentano nelle pratiche del progetto urbanistico a cavallo tra Otto e Novecento, secondo modi d'uso variabili da caso a caso. Sono modi che rivelano le loro diverse matrici, nel confronto - che è anche contesa nell'affermazione di una competenza sul progetto della città ${ }^{3}$ - tra gli apporti provenienti dall'ingegneria idraulica (l'ottimizzazione dei flussi) e sanitaria (la sanificazione, il risanamento) e le sollecitazioni dei cultori dell'arte e dell'architettura (i valori estetici, artistici, storici).

5. L'intensità e la pervasività d'impiego delle tecniche di riforma producono effetti ricompositivi differenziati. Gli interventi più ambiziosi sono appannaggio dei centri maggiori, dove più consistenti sono le risorse mobilitabili e più forti le sollecitazioni trasformative. Secondo il modello della Parigi haussmanniana, il progetto persegue, allora, una riscrittura complessiva dell'impianto urbano preesistente, ridefinito entro un sistema nuovo di relazioni. ${ }^{4}$ Spesso, però, la ristrutturazione è parziale e selettiva: agisce solo su alcuni principali capisaldi e direttrici, riorganizzando il rapporto con le nuove espansioni urbane tramite riconnessioni, nodi, recapiti e linee salienti di attraversamento. Tuttavia il più delle volte prevale la logica delle modifiche puntuali su una struttura urbana che rimane sostanzialmente invariata, con interventi di miglioramento incrementale dell'assetto esistente.

6. Capita talvolta che, in tempi relativamente ravvicinati, il medesimo luogo sia interessato da progetti di riforma che adottano impostazioni e procedimenti tecnici tra loro alternativi.

Ad esempio, Bari Vecchia dapprima $(1913)^{5}$ è oggetto di una proposta di ristrutturazione selettiva tramite l'apertura nel corpo edilizio esistente di due tracciati rettilinei (attestati sulla Cattedrale e S. Nicola), quindi $(1924)^{6}$ di un progetto di completa sostituzione secondo un impianto a maglia coerente con la città murattiana, infine $(1931)^{7}$ di un più conservativo progetto di diradamento (di Concezio Petrucci), con l'apertura di due tracciati stradali, ma di andamento irregolare e attenti ad assecondare la 'fibra' del tessuto edilizio storico.

A Bergamo, a distanza di trent'anni, si contrappongono la proposta radicale dell'ingegnere Giuseppe Chitò $(1904)^{8}$ per una «nuova strada diritta» attraverso la Città Alta e il piano di risanamento dell'ingegnere Luigi Angelini (1934), ${ }^{9}$ che Gustavo Giovannoni saluta come una delle migliori applicazioni di quella 'teoria del diradamento' da lui stesso formulata a inizio secolo. ${ }^{10}$ 
7. Nate talora come opzioni antagoniste - come il diradamento in contrapposizione allo sventramento - progressivamente le tecniche urbanistiche di riforma della città vecchia vanno a definire un repertorio di procedimenti a disposizione, da combinarsi liberamente a seconda delle diverse situazioni ed evenienze. $\mathrm{Ne}$ costituiscono un buon esempio le tavole del progetto di Alberto Morone e Fausto Natoli, che nel 1933 partecipano al Concorso per il piano regolatore della città di Como. La proposta promuove il rapporto tra città nuova e città murata attraverso un punto di cerniera su cui si innesta la direttrice prioritaria di loro interrelazione. Lungo il suo sviluppo entro la città vecchia, questo tracciato stradale principale, con scarti che seguono le quadre dell'impianto romano, è oggetto di allargamenti delle sezioni e riallineamenti dei fronti, diradamenti per creare slarghi, isolamenti per valorizzare monumenti, e veri e propri sventramenti per ridisegnare lo sbocco in una piazza a lago. ${ }^{11}$

8. All'indomani della seconda guerra mondiale il manuale di Giorgio Rigotti attualizza per l'ultima volta le tecniche di riforma. ${ }^{12}$ L'isolamento non è più l'intervento con cui stagliare emergenze architettoniche nel contesto, bensì il procedimento di scala urbana per regolare il rapporto tra città vecchia e città nuova tramite un elemento separatore - un'ampia infrastruttura viaria o una fascia a verde - da realizzarsi tutt'intorno al nucleo antico (come nel caso viennese). Dello sventramento si sottolinea il potenziale di completa riorganizzazione dell'impianto urbano originario, là dove lo si utilizzi in modo estensivo (come nel caso parigino). Del diradamento si distinguono 'diradamenti esterni', che lavorano sugli affacci stradali, risagomando lo spazio pubblico, e 'diradamenti interni', che agiscono dentro gli isolati, per svuotamento di tessuti edilizi troppo densi. Il frazionamento, infine, scompone il nucleo originario, compresso e promiscuo, in porzioni e unità - quartieri dotati di relativa autonomia - attraverso la creazione di sistemi di aree liberate, tipicamente verdi.

9. Il riutilizzo del sedime dei sistemi difensivi ormai dismessi costituisce un'opportunità eccezionale per ridefinire la relazione tra città vecchia e città nuova, in un progetto strategico di trasformazione capace di realizzare un nuovo assetto urbano. Il paradigma è il Ring di Vienna, dove un anello infrastrutturale complesso si dispone a cintura del nucleo storico sfruttando gli spazi liberati dal sistema murario e dal Glacis (la spianata prospiciente, a corona delle fortificazioni). L'anello protegge il vecchio nucleo: non intende migliorarne l'accessibilità e la permeabilità, bensì dissuaderne l'attraversamento e ridurne il congestionamento. E ridisegna il sistema dei luoghi centrali della città, riorganizzando su di sé il decentramento delle principali funzioni pubbliche, direzionali, amministrative, culturali e ricreative.

10. L’obsolescenza dei sistemi difensivi della città premoderna; le distruzioni belliche della seconda guerra mondiale; la dismissione e delocalizzazione dell'armatura produttiva della città novecentesca, che di quest'ultima aveva segnato l'impianto: per la città europea sono le tre grandi occasioni di trasformazione dal proprio interno lungo gli ultimi due secoli.

Se il caso del Ring viennese ben esemplifica le potenzialità della dismissione delle cinte murarie per un progetto di ricomposizione tra le diverse parti della città, i piani di ricostruzione in Italia - più di 400 , in relazione a centri di diverse dimensioni e a danni bellici di diversa entità ${ }^{13}$ - mostrano una gamma differenziata di interventi urbanistici di modificazione, integrazione, duplicazione, sostituzione dell'impianto urbano preesistente, in una stagione misconosciuta di 'sincretismo tecnico' del progetto urbanistico. ${ }^{14}$ Qualche decennio più tardi, l'obsolescenza delle localizzazioni produttive centrali apre a un processo di profonda ristrutturazione delle città maggiori, in una nuova proiezione metropolitana (come, ad esempio, nel Piano direttore territoriale dell'area milanese, Pim, 1991). ${ }^{15}$

11. Nel ridefinire la relazione tra città vecchia e città nuova, un'alternativa alla soluzione dell'anello è lo spostamento del centro, ovvero la sua duplicazione. Al vecchio centro urbano se ne affianca uno nuovo, destinato ad assorbirne le principali funzioni direzionali. Il trasferimento delle attività direzionali depotenzia le sollecitazioni sul centro antico, sempre più inadeguato a esse, promuovendone la salvaguardia e la riqualificazione.

All'inizio del secolo scorso, il caso di Bergamo, con il trasferimento delle funzioni terziarie di rango superiore dalla Città Alta al 'centro piacentiniano', costituisce un esempio paradigmatico di duplicazione. ${ }^{16}$ A Pechino, dove una proposta di duplicazione del centro, avanzata nel 1950 dagli urbanisti Liang Sicheng e Chen Zhanxiang non avrà seguito, ${ }^{17}$ il ribadito schema monocentrico imperniato sul nucleo originario della città getterà le premesse per il processo di profonda sostituzione del tessuto storico dei primi anni 2000.

12. L'idea di ritenere oggi i procedimenti tecnici di riforma definitivamente archiviati e inattuali non risponde al vero. Magari non più denominate come sventramenti e diradamenti, operazioni analoghe continuano a trovare applicazione, insieme alle ragioni che ne argomentano talora l'opportunità. La Rambla del Raval, nella città vecchia di Barcellona, è l'esito di un 'piano speciale di riforma interna' promosso alla fine del secolo scorso. Più in generale, nella Ciutat Vella di Barcellona per un secolo e mezzo si susseguono, realizzati o solo proposti, interventi che compongono un'antologia del progetto di riforma, in una tensione di continuo adeguamento del corpo urbano storico. ${ }^{18}$

13. Nel secondo dopoguerra, l'emergere della nozione di 'centro storico' segna un mutamento di scenario. Nei riguardi della città del passato, il progetto urbanistico abbandona il precedente consolidato atteggiamento di riforma e ne promuove uno nuovo e differente, di salvaguardia del centro storico, che riconosce a questa parte della città, considerata nella sua interezza e integrità, qualità valoriali e differenziali che la vocano alla tutela e conservazione.

14. Il fuoco del progetto urbanistico di salvaguardia si sposta dallo spazio aperto allo spazio edificato, nella combinazione di tre diversi dispositivi tecnici: usi, interventi, presidio delle qualità fisico-spaziali. La disciplina degli usi adotta una grana minuta di gran dettaglio, con indicazioni differenziate che si spingono anche all'interno dell'edificio, nelle sue diverse parti e livelli. Un secondo azzonamento modula l'intensità degli interventi ammessi nell'azione di conservazione-adeguamento-trasformazione dei singoli edifici. Un terzo dispositivo si preoccupa di disciplinare gli esiti fisici degli interventi con l'obiettivo di preservare e promuovere i fattori qualitativi del tessuto edilizio storico. 
15. Nei dispositivi di disciplina e controllo della qualità per il progetto di salvaguardia del centro storico si delineano due principali modalità e tradizioni di procedimenti tecnici. L'approccio tipologico - che ha dalla sua chiarezza, semplicità, replicabilità e facilità nel fondare una normativa - fissa e disciplina i fattori qualitativi del centro storico nei caratteri tipologici degli edifici a matrice dell'edificato. L'approccio morfologico-relazionale più complesso, eterogeneo e variegato, disponibile a molteplici diverse declinazioni contestuali - lavora, piuttosto, sulla possibilità di presidiare i fattori qualitativi del centro storico attraverso l'individuazione di unità significanti del paesaggio urbano e il riconoscimento di un vocabolario locale di elementi formali e materiali da salvaguardare.

16. Se la Carta di Gubbio del 1960 costituisce il manifesto del progetto di salvaguardia dei centri storici e dei suoi principi - segnando la distanza dalla stagione della riforma della città vecchia - il piano di Assisi di Giovanni Astengo (1958) ne sancisce un approccio 'scientifico', nell'attenta indagine e correlazione tra le condizioni del patrimonio abitativo e quelle della popolazione insediata. ${ }^{19}$ Il piano di Urbino di Giancarlo De Carlo (1964), nella sua articolata e ingegnosa disciplina per comparti, può descriversi come fondativo e rappresentativo dei procedimenti morfologico-relazionali per la disciplina del centro storico. ${ }^{20}$ Il piano del centro storico di Bologna (1969), di Pier Luigi Cervellati e Roberto Scannavini, sarà, invece, il paradigma del metodo tipologico, ${ }^{21}$ destinato a imporsi come riferimento in una pluralità di esperienze, in Italia e nel mondo. ${ }^{22}$

17. La ricerca di dispositivi progettuali per il trattamento tecnico della qualità urbana - che cosa essa sia, come si possa riconoscerla, difenderla e promuoverla - costituisce contributo essenziale della stagione della salvaguardia del centro storico. Questi meccanismi, sperimentati in quell'isola a statuto urbanistico speciale, a partire dagli anni ' 80 iniziano a travalicarne i confini: alcuni tipici procedimenti e attenzioni dapprima riservati al solo centro storico cominciano a trovare applicazione al di fuori di esso, in altre parti urbane. E si rafforza anche la convinzione che un'efficace disciplina urbanistica per le parti di qualità della città ereditata non possa che adottare forme di coinvolgimento attivo dei destinatari, in un superamento della tutela passiva. Il piano particolareggiato per Nuova Schio (1989) rivela queste caratteristiche evolutive del progetto per la città ereditata. ${ }^{23} \mathrm{Nel}$ Piano idea di Jesi (2005) prende corpo l'ipotesi di una città storica plurale e articolata per parti - non riconducibile al solo centro storico medievale e rinascimentale - e se ne promuove il ruolo urbanistico in una visione territoriale allargata. ${ }^{24}$

18. Il nuovo piano di Roma (elaborato a partire dagli anni '90 e definitivamente approvato nel 2008) sancisce il superamento teorico e metodologico della nozione di centro storico a favore del concetto di 'città storica', per un'interpretazione evolutiva del senso e del ruolo della città ereditata nel progetto urbanistico. ${ }^{25}$ 'Dal Centro Storico alla Città Storica' è il titolo di una delle tavole ufficiali di quel piano. Non più confinata entro un perimetro, non più separata dal resto della città, la città storica ne diviene infrastruttura: un sistema di elementi dalla geografia e densità spaziale variabile - esito di una selezione critica di ciò che è riconosciuto come detentore di valore e vocato alla permanenza nel palinsesto insediativo territoriale - che al pari di altri sistemi di spazi (analogamente alle infrastrutture 'grigie', 'verdi' e 'blu') si candida a svolgere un ruolo centrale nel progetto di ristrutturazione della città e di qualificazione della sua abitabilità.

19. Come nel caso di Roma, il piano di Bologna del primo decennio del 2000 adotta un approccio progettuale alla città ereditata fondato sulla nozione di 'città storica'. Nel Piano strutturale comunale (2008) e nel Regolamento urbanistico edilizio (2009) si riconoscono tre dimensioni per il progetto della città storica contemporanea: ${ }^{26}$ molecolare, areale, (infra) strutturale. Molecolare è lo sguardo volto alla definizione degli specifici profili di permanenza e persistenza dei singoli elementi e materiali urbani del palinsesto insediativo, selezionati al vaglio dell'attribuzione di valore storico. Agiscono in forma areale, per parti, quei dispositivi di disciplina urbanistica che riconoscono la geografia composita della città storica, e ne propongono un trattamento progettuale differenziato in ragione di caratteri e problemi diversificati (per cui, il centro storico è solo una delle parti di una più complessa città storica). La dimensione strutturale del progetto della città storica, infine, si esprime tipicamente nella costruzione di 'figure' che ne reinterpretano programmaticamente la presenza e il ruolo nel progetto generale di ristrutturazione della città.

20. Nel progetto della città storica contemporanea, al piano urbanistico sempre più spesso e in modo complementare ad esso si accompagnano piani e programmi ‘strategici' di azioni capaci di promuovere e combinare in un processo controllato iniziative molteplici, progetti speciali e politiche settoriali verso obiettivi convergenti di riqualificazione e rigenerazione. ${ }^{27}$

In questi programmi strategici il mix di politiche urbane coinvolte varia da caso a caso: i lavori pubblici (ed in particolare le azioni sullo spazio pubblico, ma anche quelle di re-infrastrutturazione primaria), la regolazione di settore (commercio, trasporto pubblico e sosta, mobilità/accessibilità/traffico, ricettività e pubblici esercizi, igiene e salute, cultura...) e, insieme, le opportunità offerte dalla programmazione speciale, dalla cattura di risorse su bando, dalle politiche 'per eventi', dalle misure di fiscalità locale...

21. Di nuovo in centro. Programma per la pedonalità a Bologna, predisposto all'inizio del secondo decennio degli anni 2000, costituisce un esempio di questo modo pragmatico d'operare. ${ }^{28}$ Si tratta di «un programma di coordinamento di politiche integrate» (ivi: 21) che è stato capace di un rilevante impatto sui modi di vivere e fruire gli spazi centrali di quella città. L'indice del documento che ne ha raccolto le linee d'azione le ordina e dettaglia in: accessibilità; trasporto pubblico, car sharing e veicoli elettrici; ciclabilità; sosta; riqualificazione di spazi pubblici; regole per l'uso degli spazi pubblici; valorizzazione; raccolta dei rifiuti.

22. Il caso paradigmatico è tuttavia rappresentato dal processo di rigenerazione condotto tra gli anni ' 90 e i primi 2000 nel centro storico di Genova, insieme al waterfront del Porto Vecchio. Il suo 'regista' attraverso due mandati assessorili, Bruno Gabrielli, ha definito il cosiddetto Piano della Città di Genova come un 
'piano strategico di natura operativa': una declinazione molto concreta di piano strategico che si orienta più che a una visione futuribile a un programma fattibile di azioni. ${ }^{29}$ La tensione fattiva rappresenta il carattere distintivo dell'operazione: uno strumento tradotto in schede nelle quali si esplicitano obiettivi degli interventi, risorse e loro provenienza, tempi di realizzazione, soggetti coinvolti. In questo quadro, il programma per il centro storico costituisce il cuore delle operazioni, raccolte in uno specifico documento, denominato Piano operativo del centro storico (Comune di Genova, aprile 2001).

\section{Ma, infine, perché 'tenere' la città del passato?}

A questa domanda, la prima risposta possibile parla della storia di un riconoscimento valoriale. Quello, cioè, che ci ha portato a riconoscere nella città ereditata: all'inizio, un valore estetico, artistico, architettonico di alcune sue componenti; poi, un valore storico e documentale di porzioni sempre più ampie; quindi, un valore 'di civiltà' - fissatosi nella nozione di 'bene culturale'. È la linea in cui si riflette anche l'azione dell'Unesco, nell'iscrizione di beni e siti nelle sue liste. Questo ha significato per il progetto urbanistico curarsi dapprima di singoli monumenti; poi, degli 'ambienti' in cui essi sono immersi e di cui sono parte; poi di ambienti anche privi di emergenze, 'minori', ma ugualmente meritevoli d'attenzione; infine del 'centro storico' da intendersi esso stesso, si è detto, come monumento unitario, nella sua interezza; ma poi, ancora, della 'città storica', con un'espressione che dialoga con quel 'paesaggio urbano storico' (historic urban landscape) oggetto della Raccomandazione Unesco del $2011 .^{30}$ Tuttavia proprio questo ulteriore passaggio - quello della città storica/paesaggio urbano storico - ci porta a una seconda linea di risposta alla domanda. Una risposta che si alimenta ora non di un principio valoriale contemplativo, bensì performativo e, per così dire, di utilità pratica. La città storica è - può essere un fattore d'abitabilità. I suoi materiali sono un potenziale per migliorare le prestazioni dello spazio abitabile contemporaneo. La città storica ci aiuta a vivere meglio e innerva l'urbanità della città contemporanea: è città contemporanea.

Il saggio di Ezio Micelli e Paola Pellegrini, in 'Anteprima' di questo numero, ci porta a confrontarci con questi temi, senza sconti e indulgenze.

\section{Note}

1. La forma testuale riprende un po' Dezzi Bardeschi M., 1991, Restauro: punto e da capo. Frammenti per una (impossibile) teoria, a cura di V. Locatelli. Milano: Franco Angeli.

2. Con una confezione che raccoglieva insieme il fascicolo del saggio retrospettivo di Carolina Di Biase e gli allegati 'promemoria' e 'cronologie', organizzati in sinossi di tre-più-tre manifesti pieghevoli per decenni - anni '60, '70, '80. Si veda anche il successivo: Di Biase C., 2011, «50 anni Ancsa». In: Toppetti F. (a cura di), Paesaggi e città storica. Teorie e politiche del progetto, Associazione Nazionale Centri Storico-Artistici. Firenze: Alinea.
3. Zucconi G., 1989, La città contesa. Dagli ingegneri sanitari agli urbanisti (1885-1942). Milano: Jaca Book.

4. Tamborrino R., 1998, «Haussmann e il piano per una capitale moderna». Urbanistica, 111: 116-136.

5. www.rapu.it/ricerca/jpg/Bari007.jpg (accesso: 2021.03.24).

6. www.rapu.it/ricerca/jpg/Bari009.jpg (accesso: 2021.03.24).

7. www.rapu.it/ricerca/jpg/Bari013.jpg (accesso: 2021.03.24).

8. www.rapu.it/ricerca/jpg/bergamo027.jpg (accesso: 2021.03.24).

9. www.rapu.it/ricerca/jpg/bergamo048.jpg (accesso: 2021.03.24).

10. Giovannoni G., 1943, «Una sana teoria ben applicata: il risanamento di Bergamo». Urbanistica, 3: 4-5.

11. www.rapu.it/ricerca/jpg/COMO_co29.jpg (accesso: 2021.03.24).

12. Rigotti G., 1952, Urbanistica. La composizione. Torino: UTET.

13. Cfr. Piccinato L., 1955, «Relazione generale del professor Luigi Piccinato». In: Atti del V Congresso Nazionale di Urbanistica, Urbanistica, 15-16: 27-31.

14. Bonfantini B., 2018, «I piani della ricostruzione post-bellica in Italia». In: Ferlenga A., Bassoli N. (a cura di), Ricostruzioni. Architettura, città, paesaggio nell'epoca delle distruzioni, La Triennale di Milano, catalogo della mostra. Cinisello Balsamo (Milano): Silvana Editoriale, 43-51.

15. Provincia di Milano, Centro Studi Pim, 1991, Verso la costituzione dell'area metropolitana milanese. Piano direttore territoriale provinciale: rapporto preliminare. Relazione illustrativa, Milano, giugno.

16. Bonfantini B., 2008, Bergamo. Piani 1880-2000. Santarcangelo di Romagna (Rimini): Maggioli.

17. Greco C., Santoro C., 2007, Pechino. La Città Nuova, Skira, Milano. 18. Busquets J. et al., 2004, La ciutat vella de Barcelona: un passat amb futur / The old town of Barcelona: a past with a future. Ajuntament de Barcelona, II ed.

19. [G. Astengo], 1958, «Assisi piano generale e piani particolareggiati di primo intervento». Urbanistica, 24-25.

20. De Carlo G. (1966), Urbino. La storia di una città e il piano della sua evoluzione urbanistica. Padova: Marsilio.

21. Cervellati P.L., Scannavini R., 1973, Interventi nei centri storici: Bologna. Politica e metodologia del restauro. Bologna: Il Mulino; Cervellati P.L., Scannavini R., De Angelis C., 1977, La nuova cultura delle città: la salvaguardia dei centri storici, la riappropriazione sociale degli organismi urbani e l'analisi dello sviluppo territoriale nell'esperienza di Bologna. Milano: Mondadori.

22. Cfr. Pola A.-P., 2016, a cura di, Giorgio Lombardi: l'uomo e l'architettura. Venezia: Marsilio.

23. Comune di Schio, 1990, Un manuale per 'Nuova Schio'. Piano particolareggiato per la riqualificazione urbanistica ed ambientale del quartiere operaio 'Alessandro Rossi', a cura di F. Mancuso. Venezia: Arsenale. 24. Di Giovanni A., La Palombara M., 2005, a cura di, «Jesi, un’operazione urbanistica che costruisce politiche». Urbanistica, 128: 38-79.

25. Aa.Vv., 2001, «Il nuovo piano di Roma». Urbanistica, 116: 40-286. 26. Evangelisti F., Orlandi P., Piccinini M., 2008, a cura di, La città storica contemporanea, Urban Center Bologna. Ferrara: Edisai.

27. Cfr. Storchi S., 2019, «Centro storico e città in mezzo secolo di pianificazione». In: Id. (a cura di), Centri storici e città. Parma: MUP, 24-31. 28. Comune di Bologna, 2014, Di nuovo in centro. Programma per la pedonalità a Bologna, a cura di A. Prospero con F. Evangelisti, P. Gabellini, G. Ginocchini, F. Petrei. Bologna: Urban Center.

29. Gabrielli B., Bobbio R., 2005, a cura di, «Genova, un piano strategico di natura operativa e i suoi esiti». Urbanistica, 126: 56-95.

30. Bandarin F., Van Oers R., 2014, Il paesaggio urbano storico. La gestione del patrimonio in un secolo urbano. Padova: CEDAM. 